\title{
Preparation and evaluation of polymeric microparticulates for improving cellular uptake of gemcitabine
}

This article was published in the following Dove Press journal:

International Journal of Nanomedicine

5 May 2012

Number of times this article has been viewed

\author{
Ji-Ho Lim ${ }^{1, *}$ \\ Sung-Kyun You',* \\ Jong-Suep Baek' \\ Chan-Ju Hwang' \\ Young-Guk $\mathrm{Na}^{\prime}$ \\ Sang-Chul Shin ${ }^{2}$ \\ Cheong-Weon Cho' \\ 'College of Pharmacy and Institute \\ of Drug Research and Development, \\ Chungnam National University, \\ Gungdong, Yuseonggu, Daejeon, \\ South Korea, ${ }^{2}$ College of Pharmacy, \\ Chonnam National University, Buggu, \\ Gwangju, South Korea \\ *These authors contributed equally \\ to this work
}

Background: Gemcitabine must be administered at high doses to elicit the required therapeutic response because of its very short plasma half-life due to rapid metabolism. These high doses can have severe adverse effects.

Methods: In this study, polymeric microparticulate systems of gemcitabine were prepared using chitosan as a mucoadhesive polymer and Eudragit L100-55 as an enteric copolymer. The physicochemical and biopharmaceutical properties of the resulting systems were then evaluated.

Results: There was no endothermic peak for gemcitabine in any of the polymeric gemcitabine microparticulate systems, suggesting that gemcitabine was bound to chitosan and Eudragit L100-55 and its crystallinity was changed into an amorphous form. The polymeric gemcitabine microparticulate system showed more than $80 \%$ release of gemcitabine in 30 minutes in simulated intestinal fluid. When mucin particles were incubated with gemcitabine polymeric microparticulates, the zeta potential of the mucin particles was increased to $1.57 \mathrm{mV}$, indicating that the polymeric gemcitabine microparticulates were attached to the mucin particles. Furthermore, the F53 polymeric gemcitabine microparticulates having $150 \mathrm{mg}$ of chitosan showed a 3.8-fold increased uptake of gemcitabine into Caco-2 cells over 72 hours compared with gemcitabine solution alone.

Conclusion: Overall, these results suggest that polymeric gemcitabine microparticulate systems could be used as carriers to help oral absorption of gemcitabine.

Keywords: gemcitabine, polymeric microparticulates, mucoadhesive, enteric coating, cellular uptake, oral absorption

\section{Introduction}

Gemcitabine hydrochloride $\left(2^{\prime}, 2^{\prime}\right.$-difluoro-2'deoxycytidine hydrochloride) has therapeutic activity against a variety of solid malignancies, including colon, lung, pancreatic, breast, bladder and ovarian cancers. ${ }^{1-4}$ Once transported into the cell, gemcitabine must be phosphorylated by deoxycytidine kinase into its active form, 5 -triphosphategemcitabine, which is incorporated into the DNA strand, halting elongation and causing cell death. Gemcitabine action also involves ribonucleotide reductase inhibition. ${ }^{5}$ However, gemcitabine (pKa 3.58) is rapidly metabolized in the blood, liver, and kidneys by cytidine deaminase into $2^{\prime}, 2^{\prime}$-difluoro- 2 '-deoxyuridine, an inactive derivative. ${ }^{6}$ Gemcitabine has a very short plasma half-life when administered intravenously, which is a major limitation of this anticancer compound. ${ }^{7}$ Recently, it has been postulated that continuous treatment with oral gemcitabine might be efficacious in human malignancies, and that oral dosing would be more convenient for patients than intravenous administration. Oral gemcitabine at low dose levels is
Correspondence: Cheong-Weon Cho College of Pharmacy, Chungnam, National University, Gungdong, Yuseonggu, Daejeon, South Korea Tel +82428215934

Fax +82 428236566

Email chocw@cnu.ac.kr 
well tolerated and the main pattern of toxicity observed is gastrointestinal, whereas very little hematologic toxicity is observed, consistent with the low systemic exposure of gemcitabine. ${ }^{8}$ This is due to extensive first-pass metabolism to $2^{\prime}, 2^{\prime}$-difluoro-2'-deoxyuridine, which has been reported to be present mainly in the liver in humans and the kidney in mice. ${ }^{9}$ Thus, oral alternatives to intravenous gemcitabine administration can be investigated by strategies that facilitate enhanced intestinal absorption and circumvent metabolic inactivation by cytidine deaminase in the liver. ${ }^{10}$ Some type of protection for the anticancer drug, gemcitabine, which undergoes rapid metabolism in the plasma, must be developed. Recently, several approaches have been attempted to decrease deamination of gemcitabine to $2^{\prime}, 2^{\prime}$-difluoro- $2^{\prime}$ deoxyuridine, such as coupling a long chain fatty acid or an isoprenoid chain of squalene to the terminal amino group of gemcitabine, thereby protecting it from deamination by cytidine deaminase. ${ }^{11-13}$

On the other hand, colloidal drug delivery systems have received increasing attention as a possible means to obtain a higher therapeutic effect, lower toxicity, higher intestinal uptake, and protection from in vivo metabolism of incorporated drugs. ${ }^{1}$ From a structural point of view, nanoparticles comprise different systems, including nanospheres and nanocapsules. ${ }^{2}$

A high dose of gemcitabine is needed because rapid metabolism induces a very short half-life and low oral bioavailability $(9.5 \%){ }^{14,15}$ In this work, we prepared polymeric particulate systems of gemcitabine using chitosan and polymethacrylate polymers to develop an oral delivery system and evaluated its physicochemical characteristics. Chitosan confers the polymeric particulate systems with mucoadhesion, and Eudragit L100-55, a polymethacrylate polymer, makes them enteric-coated to prevent gastric irritation.

\section{Materials and methods Materials}

Gemcitabine hydrochloride was obtained from Dalian Wista Pharma Co, Ltd (Dalian, China). Polyvinyl alcohol (molecular weight 146-186,000), mannitol, and mucin were purchased from Sigma (Steinheim, Switzerland). Eudragit L100-55 [poly(methacrylic acid, ethyl acrylate) 1:1] was acquired from Degussa (Frankfurt, Germany). Chitosan (molecular weight 5-10,000) was purchased from Chitolife Co, Ltd (Pyungtaek, Korea). Sodium chloride was purchased from Samchun Chemical Co, Ltd (Pyungtaek). Dulbecco's modified Eagle's medium, fetal bovine serum, nonessential amino acid solution $(10 \mathrm{mM}, 100 \times)$, penicillin-streptomycin solution $(10,000 \mathrm{U} / \mathrm{mL}$ penicillin and $10 \mathrm{mg} / \mathrm{mL}$ of streptomycin), trypsin-EDTA solution $(0.05 \%$ trypsin, $0.53 \mathrm{mM}$ EDTA), N-(2-hydroxyethyl)piperazine-N'-2-ethane-sulfonic acid, and Hank's Balanced Salt Solution with calcium and magnesium (without phenol red) were purchased from Gibco Laboratories (Cergy Pontoise, France). All other chemicals and solvents were of analytical reagent grade and used without further purification.

\section{Cell cultures}

Caco-2 cells were purchased from the Korean Cell Line Bank (Seoul, Korea). Caco-2 cells (passage number 46-52) were cultured in Dulbecco's modified Eagle's medium supplemented with $10 \%$ fetal bovine serum, $1 \%$ nonessential amino acid solution, 100 units $/ \mathrm{mL}$ penicillin, and $0.1 \mathrm{mg} / \mathrm{mL}$ streptomycin in a 5\% $\mathrm{CO}_{2}$ atmosphere with $95 \%$ humidity in a $37^{\circ} \mathrm{C}$ incubator.

\section{Preparation of polymeric gemcitabine microparticulates}

Polymeric gemcitabine microparticulates were produced using a multiple emulsion method ${ }^{16,17}$ involving emulsification of an aqueous solution of the drug with a polymer solution in an organic solvent to form a w/o emulsion. This emulsion was further emulsified into a stirred aqueous processing medium containing an emulsion stabilizer to form a w/o/w emulsion, and the $\mathrm{pH}$ of each individual reaction solution was also measured. The organic solvent was removed over one hour by rotary evaporation (RE111 Rotavapor, Buchi, Switzerland). Briefly, $50 \mathrm{mg}$ of gemcitabine was dissolved in $1 \mathrm{~mL}$ of water and then mixed well with solubilized chitosan. At the same time, $500 \mathrm{mg}$ of Eudragit L100-55 was dissolved in $10 \mathrm{~mL}$ of $\mathrm{n}$-butanol. Gemcitabine including chitosan was slowly added to the organic Eudragit L100-55 solution and homogenized at 12,000 rpm for 10 minutes using a homogenizer (Ultra-Turrax T25, IkaWerk, Staufe, Germany) to form the primary emulsion $\left(\mathrm{w}_{1} / \mathrm{o}\right)$. The $\mathrm{w}_{1} / \mathrm{o}$ emulsion was further emulsified in $10 \mathrm{~mL}$ of a $1.0 \%$ aqueous solution of polyvinyl alcohol and $0.9 \%$ sodium chloride as an emulsion stabilizer at $5000 \mathrm{rpm}$ for 10 minutes using a homogenizer, resulting in formation of a $\mathrm{w}_{1} / \mathrm{o} / \mathrm{w}_{2}$ emulsion. These emulsions were rotary-evaporated to eliminate the organic phase, leaving hardened particles in an aqueous medium ${ }^{18}$ and then centrifuged at $3000 \mathrm{rpm}$ for 10 minutes. Finally, $5 \mathrm{~mL}$ of $10 \%$ mannitol solution was added and the mixture was lyophilized. In this experiment, polymeric gemcitabine microparticulates containing chitosan were called F49-F53 according to the increase in amount of chitosan, ie, $10 \mathrm{mg}, 25 \mathrm{mg}, 50 \mathrm{mg}$, 
$100 \mathrm{mg}$, or $150 \mathrm{mg}$. The polymeric gemcitabine microparticulates without chitosan (F10) and the physical mixture of individual components of gemcitabine, chitosan, and Eudragit L100-55 were prepared as controls.

\section{Assay of polymeric gemcitabine microparticulate}

Polymeric gemcitabine microparticulates $(10 \mathrm{mg})$ were dissolved in $10 \mathrm{~mL}$ of sodium phosphate buffer and methanol $(9: 1, v / v)$, sonicated for 10 minutes, and then centrifuged at $3000 \mathrm{rpm}$ for 10 minutes. After centrifugation, $1 \mathrm{~mL}$ of supernatant was filtered through a membrane filter with a pore size of $0.2 \mu \mathrm{m}$ and analyzed by high-pressure liquid chromatography. An Agilent 1100 liquid chromatography system with an autosampler and ultraviolet detector were used. A C18 column $(4.6 \times 250 \mathrm{~mm}, 5 \mu \mathrm{m}$ particle size Eclipse $^{\circledR}$, Agilent Technologies, Santa Clara, CA) was used. The flow rate of the mobile phase was $1.2 \mathrm{~mL} /$ minute and the detection wavelength was set to $275 \mathrm{~nm}$. The mobile phase was a mixture of $97 \%$ monosodium phosphate buffer and $3 \%$ methanol. The column temperature was maintained at $35^{\circ} \mathrm{C}$. The amount of gemcitabine in the polymeric microparticulates was determined by examining the drug loading and entrapment efficiency. The percentage drug loading was calculated by multiplying 100 by the ratio of total amount of drug extracted from the polymeric matrix of a known weight of microparticulates to the total weight of the microparticulates used before extraction. The encapsulation efficiency was calculated by multiplying 100 by the ratio of weight of drug present in a batch of microparticulates to the weight of drug used in the formulation.

\section{Physicochemical characterization of polymeric gemcitabine microparticulates} The morphology and surface characteristics of the gemcitabine polymeric microparticulates were examined by scanning electron microscopy (Model JEOL, JSM-7000F, Japan) operating at an accelerating voltage of $20 \mathrm{kV}$. The material was mounted on aluminum holders with carbonconducting glue and coated with a $20 \mathrm{~nm}$ gold layer in a Balzer's MED 010 sputtering device. The particle size and zeta potential of the polymeric gemcitabine microparticulates were determined by dynamic laser scattering (ELS8000 particle size analyzer, Otasuka Electronics, Japan). Differential scanning calorimetry was performed using a Perkin-Elmer DSC-7 instrument (SC S-650, Scinco, Korea) and Pyris software (version 5). The temperature axis and cell constant were calibrated using indium. A weighed sample
(1.8 mg) of gemcitabine or polymeric gemcitabine microparticulates in a pin-holed aluminum pan was heated from $20^{\circ} \mathrm{C}$ to $400^{\circ} \mathrm{C}$ at a rate of $20^{\circ} \mathrm{C}$ per minute.

\section{In vitro gemcitabine release profiles}

A weighed amount of polymeric microparticulates $(50 \mathrm{mg}$ ) containing $2.5 \mathrm{mg}$ of gemcitabine based on entrapment efficiency data was dissolved in $100 \mathrm{~mL}$ of simulated gastric fluid or simulated intestinal fluid as the release medium using a paddle dissolution tester (Labfine Scientific Instruments, Anyang, Korea) based on Korean Pharmacopeia (KP) IX. At a predetermined time, $1 \mathrm{~mL}$ samples were withdrawn and replaced with the same volume of fresh medium adjusted to $37^{\circ} \mathrm{C}$. The samples were filtered through a membrane filter with a pore size of $0.2 \mu \mathrm{m}$ and analyzed by high-pressure liquid chromatography.

\section{Mucoadhesion test}

We also evaluated the mucoadhesion forces of the polymeric gemcitabine microparticulates. The mucin particle method ${ }^{19}$ is a simple mucoadhesion test in which commercial mucin particles are used. Mucoadhesion was examined by measuring the change in zeta potential of the mucin particles in a certain concentration of polymeric gemcitabine microparticulates. Briefly, mucin particles were suspended in distilled water at a concentration of $1 \% \mathrm{w} / \mathrm{v}$ and then dissolved in an appropriate amount of polymeric gemcitabine microparticulates in simulated intestinal fluid at room temperature. After incubation for 12 hours, the zeta potential was measured.

\section{Cytotoxicity assay}

After a 72-hour incubation of the cells (70\% confluent) with gemcitabine, cytotoxicity was determined by MTT assay according to the manufacturer's protocol. Briefly, after incubation of cells with gemcitabine, MTT (3-(4,5dimethylthiazol-2yl)-2,5-diphenyl-2H-tetrazolium bromide) was added to each well and incubated for 2 hours at $37^{\circ} \mathrm{C}$. The crystals of viable cells were solubilized in isopropanol. Absorbance was determined at $570 \mathrm{~nm}$ in a microplate reader (Sunrise, Tecan, Austria). Cell viability (\%) was represented with the (optical density [OD] of samples-treated cells divided by OD of cells incubated without samples $) \times 100$.

\section{Cellular uptake study}

One day before the uptake experiments, Caco- 2 cells (passage number 46-52) were seeded in 24-well plates at a density of $5 \times 10^{5}$ cells/well. The cells were washed twice with serumfree Dulbecco's modified Eagle's medium and then exposed 
to either $10 \mathrm{mM}$ of gemcitabine solution or polymeric gemcitabine microparticulates for 72 hours. The cellular uptake studies were subsequently terminated by aspirating the medium and washing the cells three times with phosphatebuffered saline. The cells were then lysed with $0.2 \mathrm{~N} \mathrm{NaOH}$, sonicated for 10 minutes, and then centrifuged at $3000 \mathrm{rpm}$ for 10 minutes. The supernatant was filtered through a membrane filter with a pore size of $0.2 \mu \mathrm{m}$ and analyzed by high-pressure liquid chromatography. The amount of protein in each sample was determined using the bicinchoninic acid method (Sigma, Steinheim, Switzerland).

\section{Statistical analysis}

The Student's $t$-test was used to compare groups. A $P$ value $<0.05$ was considered statistically significant.

\section{Results and discussion Surface morphology}

The polymeric gemcitabine microparticulates using various amounts of chitosan showed a round morphology (Figure 1).

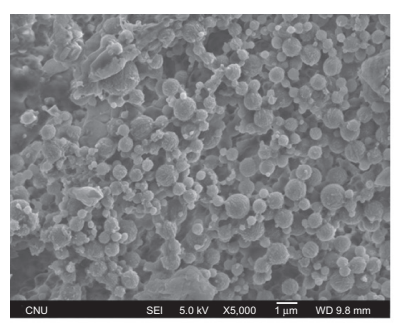

F10

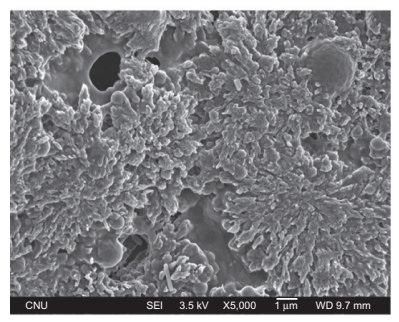

F50

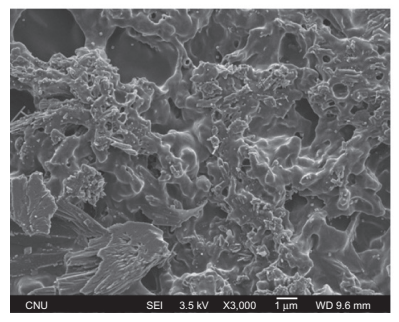

F52

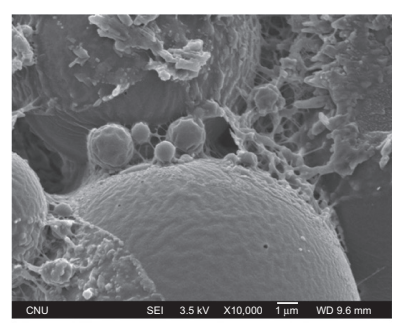

F49

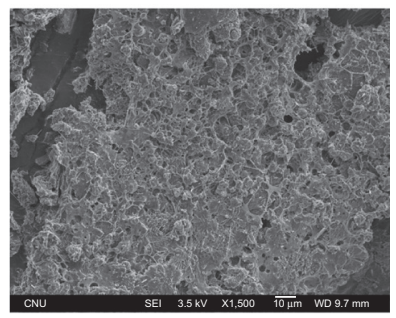

F51

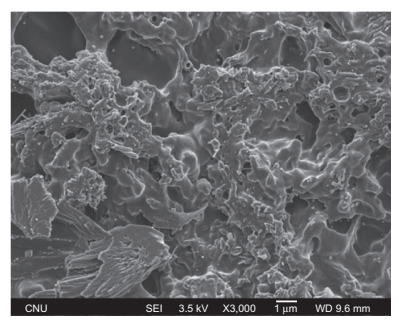

F53
Figure I Scanning electron micrographs of polymeric gemcitabine microparticulates. Notes: FIO is polymeric gemcitabine microparticulates without chitosan and F49F53 is polymeric gemcitabine microparticulates according to the increase in chitosan amount, ie, $10 \mathrm{mg}, 25 \mathrm{mg}, 50 \mathrm{mg}, 100 \mathrm{mg}$, or $150 \mathrm{mg}$.
Some smooth spherical microparticulates were self-aggregated and showed a sponge-like shape that might be attributed to the extrusion of aqueous internal droplets from the polymeric matrix to the external aqueous region during particle formation because of the relative instability of the quasi w/o emulsion. ${ }^{20-22}$ The particles were not only irregular balls with uneven surfaces, but also showed high surface aggregation forms. The amount of chitosan had no effect on the size and shape of the polymeric gemcitabine microparticulates.

\section{Differential scanning calorimetry study}

In order to assess the changes in the solid state of the polymeric gemcitabine microparticulates, differential scanning calorimetry analysis was performed. The thermal curve of gemcitabine was typical of a crystalline substance, with an endothermic peak at around $280.69^{\circ} \mathrm{C}$ (Figure 2). An endothermic peak of gemcitabine was observed in the physical mixture, but there was no endothermic peak of gemcitabine in the polymeric gemcitabine microparticulates, suggesting that gemcitabine was chemically bound to chitosan and Eudragit L100-55, and subsequently its crystallinity had changed into the amorphous form. Furthermore, in the case of F10 without chitosan, there was no endothermic peak of gemcitabine. These results implied that Eudragit L100-55, which is a polyelectrolyte, played an important role in the transformation of gemcitabine by electrostatic interaction into an amorphous form.

\section{Particle size and zeta potential}

The particle size and zeta potential of polymeric gemcitabine microparticulates were evaluated to determine their physicochemical properties. In particular, as the amount of chitosan was increased from $10 \mathrm{mg}$ to $150 \mathrm{mg}$, the zeta potential values for all the polymeric gemcitabine microparticulates except for F10 showed a tendency to increase (Figure 3). These data were consistent with those reported previously, ${ }^{23,24}$ suggesting that gemcitabine and chitosan were electrostatically complexed during particle preparation and that the particles became aggregated. The $\mathrm{pH}$ of the gemcitabine aqueous solution was 2.1 and that of the gemcitabine aqueous solution and solubilized chitosan mixture was 2.4. After Eudragit L100-55 solution with a $\mathrm{pH}$ of 1.75 was added to the above mixture, the $\mathrm{pH}$ became 2.3. Subsequently, after polyvinyl alcohol solution having a $\mathrm{pH}$ of 5.3 was added to the $\mathrm{w}_{1} / \mathrm{o}$ emulsion, the $\mathrm{pH}$ of the final $\mathrm{w}_{1} / \mathrm{o} / \mathrm{w}_{2}$ was 3.1 . The zeta potential of gemcitabine itself was $0.95 \pm 0.37 \mathrm{mV}$ and that of chitosan was $6.1 \pm 0.30 \mathrm{mV}$. The zeta potential of the polymeric gemcitabine microparticulates without chitosan 


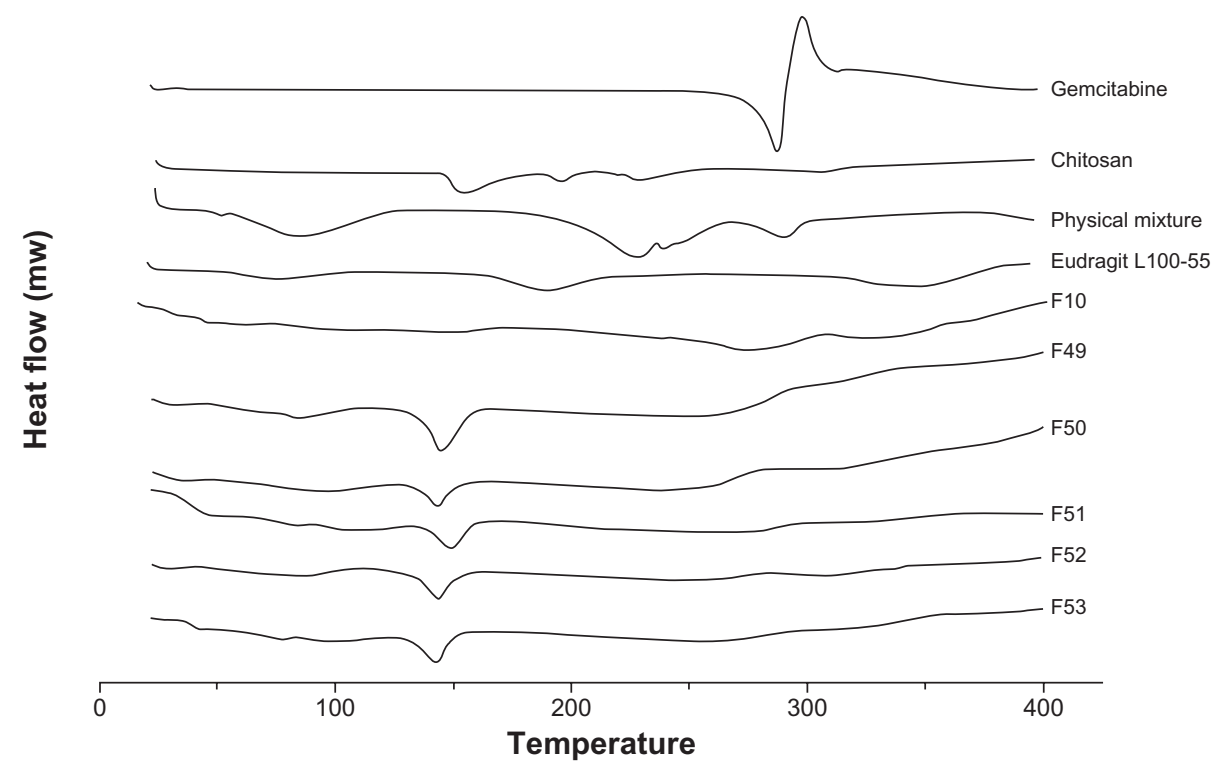

Figure 2 Differential scanning calorimetric thermograms of polymeric gemcitabine microparticulates.

Notes: The differential scanning calorimetric runs were conducted at $20^{\circ} \mathrm{C}-400^{\circ} \mathrm{C}$ and a rate of $20^{\circ} \mathrm{C}$ per minute. $\mathrm{Fl} 0$ is polymeric gemcitabine microparticulates without chitosan and F49-F53 is polymeric gemcitabine microparticulates according to the increase in chitosan amount, ie, $10 \mathrm{mg}, 25 \mathrm{mg}, 50 \mathrm{mg}, 100 \mathrm{mg}$, or $150 \mathrm{mg}$.

(F10) was $-16.7 \pm 1.82 \mathrm{mV}$, suggesting that gemcitabine and Eudragit L100-55 solutions were strongly repulsed, and subsequently showed the fewest microparticulates.

\section{Assay of gemcitabine polymeric microparticulates}

The concentration of gemcitabine in the polymeric microparticulates was quantified by measuring the yield, drug loading, and entrapment efficiency of gemcitabine. Fifty milligrams of polymeric gemcitabine microparticulates were assayed and entrapment efficiency was calculated. Poor entrapment of alendronate sodium in poly(lactide-co-glycolide) microspheres prepared by $\mathrm{w} / \mathrm{o} / \mathrm{w}$ emulsification has been reported previously. ${ }^{25}$ It was suggested that because alendronate sodium was water-soluble $(10 \mathrm{mg} / \mathrm{mL})$, it had a tendency to escape to the external aqueous phase during microsphere formation and during washing of the formed microspheres. Therefore, drug hydrophilicity is a major challenge in the development of microspheres with high entrapment efficiency. ${ }^{22,26,27}$ It was found that when the drug loading was lowered, entrapment efficiency was also lowered. Except when no chitosan was present or a low chitosan dose of $10 \mathrm{mg}$ was present in the polymeric microparticulates, drug loading was determined to be $4.5 \%-5.8 \%$ and entrapment efficiency was determined to be $88.7 \%-97.9 \%$ (Table 1). However, there was no significant relationship

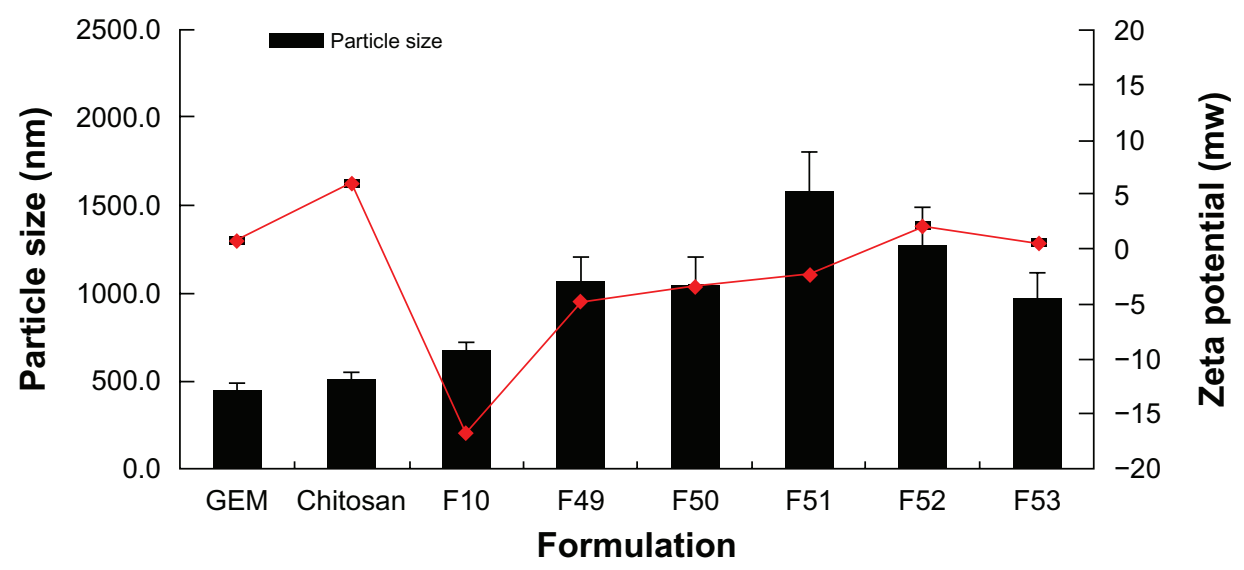

Figure 3 Particle size and zeta potential of polymeric gemcitabine microparticulates according to the amount of chitosan.

Notes: FIO is polymeric gemcitabine microparticulates without chitosan and F49-F53 is gemcitabine polymeric microparticulates according to the increase in chitosan amount, ie, $10 \mathrm{mg}, 25 \mathrm{mg}, 50 \mathrm{mg}, 100 \mathrm{mg}$, or $150 \mathrm{mg}$. 
Table I Formulation of polymeric gemcitabine microparticulates with chitosan

\begin{tabular}{lllll}
\hline $\begin{array}{l}\text { Formula } \\
\text { number }\end{array}$ & $\begin{array}{l}\text { Chitosan } \\
(\mathbf{m g})\end{array}$ & $\begin{array}{l}\text { Yield } \\
\mathbf{( \% )}\end{array}$ & $\begin{array}{l}\text { Drug } \\
\text { loading (\%) }\end{array}$ & $\begin{array}{l}\text { Entrapment } \\
\text { efficiency (\%) }\end{array}$ \\
\hline F10 & 0 & 86.4 & 1.8 & 33.4 \\
F49 & 10 & 86.4 & 1.7 & 33.9 \\
F50 & 25 & 91.7 & 5.8 & 93.7 \\
F51 & 50 & 80.8 & 5.0 & 88.7 \\
F52 & 100 & 100.8 & 4.5 & 97.9 \\
F53 & 150 & 99.5 & 4.7 & 93.0 \\
\hline
\end{tabular}

between amount of chitosan and drug loading and entrapment efficiency. Jang et $\mathrm{al}^{28}$ reported that when the solvent evaporation rate was increased from $20 \mathrm{rpm}$ to $280 \mathrm{rpm}$, the entrapment efficiency increased from $28.8 \% \pm 3.3 \%$ to $52.5 \% \pm 6.8 \%$, inferring that rapid membrane formation is an important phenomenon, preventing leakage of salmon calcitonin to the outer water phase. Also, the salmon calcitonin diffusion-delaying effect of high molecular weight polyvinyl alcohol $(86,000)$ was greater than that of low molecular weight polyvinyl alcohol $(66,000)$, which resulted in higher salmon calcitonin entrapment efficiency. In this study, the authors used high molecular weight polyvinyl alcohol $(146,000-186,000)$ and increased the solvent evaporation rate to obtain higher entrapment efficiency (Table 1).

\section{In vitro gemcitabine release profiles}

There was less than $10 \%$ release of gemcitabine from the polymeric microparticulates after 2 hours in simulated gastric fluid, due to enteric coating of the gemcitabine primary emulsion by Eudragit L100-55 (data not shown). In contrast, the polymeric gemcitabine microparticulates showed more than $80 \%$ release of gemcitabine in 30 minutes in simulated intestinal fluid (Figure 4). Rapid release of gemcitabine might occur due to fast dissolving of Eudragit L100-55 in simulated intestinal fluid. However, there was no relationship between

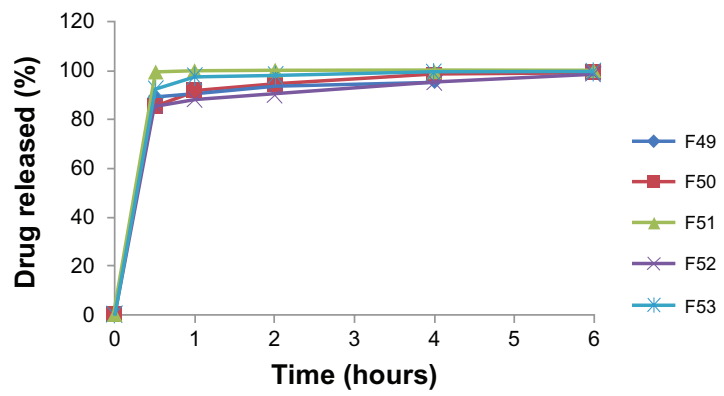

Figure 4 In vitro release profile of gemcitabine from polymeric gemcitabine microparticulates in simulated intestinal juices.

Notes: F49-F53 is polymeric gemcitabine microparticulates according to the increase of chitosan amount, ie, $10 \mathrm{mg}, 25 \mathrm{mg}, 50 \mathrm{mg}, 100 \mathrm{mg}$, or $150 \mathrm{mg}$. the percentage of gemcitabine released and the amount of chitosan.

\section{Mucoadhesion test}

The mucoadhesion of polymeric gemcitabine microparticulates was evaluated using commercially available porcine mucin particles in a simple mucoadhesion test. The surface properties of the mucin particles were expected to change due to adhesion of the polymeric gemcitabine microparticulates if the microparticulates had mucoadhesive properties. After the mucin particle suspensions were mixed with polymeric gemcitabine microparticulates dissolved in simulated intestinal fluid to remove the enteric coating of Eudragit L100-55, the zeta potential of the mucin particles was measured (Figure 5). This polymer has mucoadhesive properties due to its positive charges at neutral $\mathrm{pH}$ that enable electrostatic interaction with mucus or a negatively charged mucosal surface. ${ }^{19}$ The zeta potential of $1 \%$ mucin particle suspensions alone ( $\mathrm{pH} 4.1$ ) was $-16.14 \pm 2.75 \mathrm{mV}$ and that of $1 \%$ mucin particle suspensions when incubated with $10 \mathrm{mg}$ of chitosan was $-1.17 \pm 0.32 \mathrm{mV}$, suggesting that the zeta potential was increased due to adhesion of chitosan to the mucin particles. Therefore, the negative charge of the mucin particles appeared to be neutralized by the positive charge of chitosan absorbed onto their surface. When the amount of chitosan was increased from $10 \mathrm{mg}$ to $150 \mathrm{mg}$, the zeta potential increased from $-22.14 \pm 2.50 \mathrm{mV}$ to $-1.22 \pm 0.18 \mathrm{mV}$. As a reference, the zeta potential of F10 to mucin particles was $-4.52 \pm 1.67 \mathrm{mV}$.

\section{Cytotoxicity and cellular uptake}

Before the cellular uptake study of the polymeric gemcitabine microparticulates, a cytotoxicity study of gemcitabine against Caco- 2 cells was performed to determine the $\mathrm{IC}_{50}$ of gemcitabine. Gemcitabine showed cytotoxicity at concentrations above $50 \mathrm{mM}$ in Caco-2 cells. The cell viability dropped

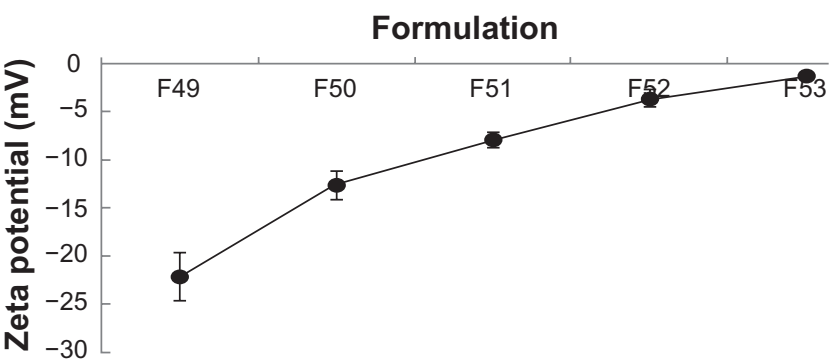

Figure 5 Zeta potential after incubation of polymeric gemcitabine microparticulates with mucin particles.

Notes: FIO is polymeric gemcitabine microparticulates without chitosan and F49F53 is polymeric gemcitabine microparticulates according to the increase in chitosan amount, ie, $10 \mathrm{mg}, 25 \mathrm{mg}, 50 \mathrm{mg}, 100 \mathrm{mg}$, or $150 \mathrm{mg}$. 
suddenly at a gemcitabine concentration of 70-100 mM in Caco-2 cells, and subsequently the $\mathrm{IC}_{50}$ of gemcitabine against Caco-2 cells was determined to be $52.4 \mathrm{mM}$ (Figure $6 \mathrm{~A}$ ). Therefore, gemcitabine solution or polymeric gemcitabine microparticulates corresponding to a gemcitabine $10 \mathrm{mM}$ was added to each well and the percentage of gemcitabine uptake into Caco-2 cells was measured (Figure $6 \mathrm{~B}$ ). The gemcitabine solution showed $8.65 \% \pm 3.64 \%$ intestinal cellular uptake of gemcitabine into Caco-2 cells over 72 hours because gemcitabine was released from the polymeric microparticulates in the cell culture medium $(\mathrm{pH}$ 7.85). In particular, polymeric gemcitabine microparticulates containing $150 \mathrm{mg}$ of chitosan (F53) showed a 3.8-fold increase in intestinal cellular uptake of gemcitabine into Caco-2 cells over 72 hours compared with uptake of gemcitabine solution. As the amount of chitosan increased, the zeta potential values (Figure 3) and cellular uptake of gemcitabine polymeric microparticulates also increased. It has previously been suggested that chitosan has mucoadhesive properties, as shown in Figure 5, and improves permeation of gemcitabine through Caco-2 cells. ${ }^{24}$ This is consistent with a report showing that microparticles prepared with chitosan and glyceryl monooleate exhibited mucoadhesive properties and a four-fold increase in cellular uptake. ${ }^{29}$ Chitosan
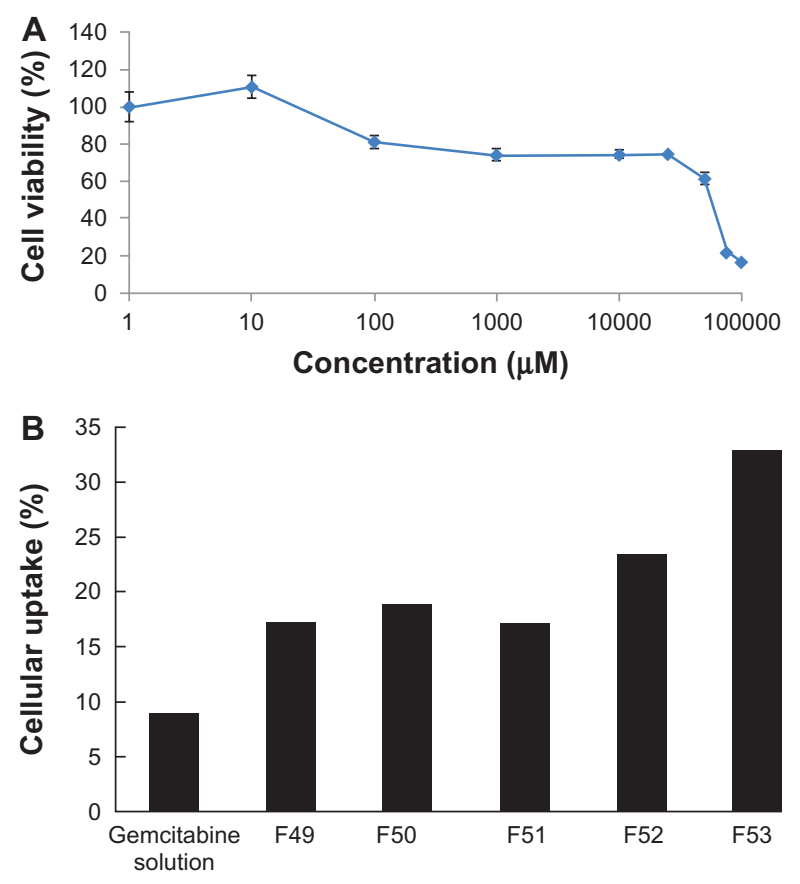

Figure 6 Cytotoxicity of gemcitabine according to the increase of gemcitabine concentration against Caco-2 cells (A) and cellular uptake of polymeric gemcitabine microparticulates into Caco-2 cells (B).

Notes: FIO is polymeric gemcitabine microparticulates without chitosan and F49F53 is polymeric gemcitabine microparticulates according to the increase of chitosan amount, ie, $10 \mathrm{mg}, 25 \mathrm{mg}, 50 \mathrm{mg}, 100 \mathrm{mg}$, or $150 \mathrm{mg}$. is a hydrophilic, biodegradable, biocompatible, positively charged polysaccharide with low toxicity that in recent years has found applications in cosmetic, biotechnology, and drug delivery systems. The relationship between mucoadhesiveness of polymeric gemcitabine microparticulates in mucin particles with cellular uptake of gemcitabine from polymeric gemcitabine microparticulates into Caco-2 cells could not be explained because Caco- 2 cells do not secrete mucin. Also, it could not be distinguished whether polymeric gemcitabine microparticulates were actually internalized across Caco-2 cells or were bound onto the Caco-2 cell surface due to the mucoadhesive properties of chitosan. The authors recently reported that formulation of alendronate microparticles including chitosan showed a three-fold increase in uptake of alendronate into Caco- 2 cells and caused a significant $42.4 \%$ enhancement of alendronate permeability across Caco- 2 monolayers. ${ }^{24}$ Therefore, further studies on the internalization of polymeric gemcitabine microparticulates and their absorption in vivo will be performed.

\section{Conclusion}

Polymeric microparticulate systems containing gemcitabine were prepared using various amounts of chitosan as a mucoadhesive polymer and Eudragit L100-55 as an enteric copolymer. F53 polymeric gemcitabine microparticulates showed a 3.8-fold increase in uptake of gemcitabine into Caco-2 cells over 72 hours compared with uptake of gemcitabine solution. Overall, these results suggest that polymeric microparticulate systems could improve the cellular uptake of gemcitabine.

\section{Acknowledgment}

This work was supported by the Priority Research Centers Program (2009-0093815) through the National Research Foundation of Korea funded by the Ministry of Education, Science, and Technology.

\section{Disclosure}

The authors report no conflicts of interest in this work.

\section{References}

1. Moghimi S, Hunter A, Murray J. Long-circulating and target-specific nanoparticles: theory to practice. Pharmacol Rev. 2001;53:283-318.

2. Couvreur P, Barratt G, Fattal E, et al. Nanocapsule technology: a review. Crit Rev Ther Drug Carrier Syst. 2002;19:99-134.

3. Vauthier C, Dubernet C, Fattal E, et al. Poly(alkylcyanoacrylates) as biodegradable materials for biomedical applications. Adv Drug Deliv Rev. 2003;55:519-548.

4. Hertel L, Boder G, Kroin J, et al. Evaluation of the antitumor activity of gemcitabine (2',2'-difluoro-2'-deoxycytidine). Cancer Res. 1990;50:4417-4422. 
5. Plunkett W, Huang P, Xu Y, et al. Gemcitabine: metabolism, mechanism of action, and self potentiation. Semin Oncol. 1995;22:3-10.

6. Heinemann V, Xu Y, Chubb S, et al. Cellular elimination of 2', 2'-difluorodeoxycytidine 5'-triphosphate: a mechanism of self-potentiation. Cancer Res. 1992;52:533-539.

7. Abbruzzese J, Grunewald R, Weeks E, et al. A Phase I clinical, plasma, and cellular pharmacology study of gemcitabine. J Clin Oncol. 1991;9:491-498.

8. Veltkamp S, Jansen R, Callies S, et al. Oral administration of gemcitabine in patients with refractory tumors: a clinical and pharmacologic study. Clin Cancer Res. 2008;14:3477-3486.

9. Camiener G, Smith C. Studies of the enzymatic deamination of cytosine arabinoside. I, enzyme distribution and species specificity. Biochem Pharmacol. 1965;14:1405-1416.

10. Song X, Lorenzi PL, Landowski CP, et al. Amino acid ester prodrugs of the anticancer agent gemcitabine: Synthesis, bioconversion, metabolic bioevasion, and hPEPT1-mediated transport. Mol Pharm. 2005;2:157-167.

11. Castelli F, Sarpietro MG, Ceruti M, et al. Characterization of lipophilic gemcitabine prodrug-liposomal membrane interaction by differential scanning calorimetry. Mol Pharmacol. 2006;3:737-744.

12. Couvreur P, Stella B, Reddy L, et al. Squalenoyl nanomedicines as potential therapeutics. Nano Lett. 2006;6:2544-2548.

13. Stella B, Arpicco S, Rocco F, et al. Encapsulation of gemcitabine lipophilic derivatives into polycyanoacrylate nanospheres and nanocapsules. Int J Pharm. 2007;344:71-77.

14. Matsuda A, Sasaki T. Antitumor activity of sugar-modified cytosine nucleosides. Cancer Sci. 2004;95:105-111.

15. Reddy LH, Couvreur P. Novel approaches to deliver gemcitabine to cancers. Curr Pharm Des. 2008;14:1124-1137.

16. Ehtezazi T, Washington C, Melia C. Determination of the internal morphology of poly(D,L-lactide) microspheres using stereological methods. J Control Release. 1999;57:301-314.

17. Ehtezazi T, Washington C. Controlled release of macromolecules from PLA microspheres using porous structure topology. J Control Release. 2000;68:361-372.
18. Mora-Huertas C, Fessi H, Elaissari A. Polymer-based nanocapsules for drug delivery. Int J Pharm. 2009;385:113-142.

19. Takeuchi H, Thongborisute J, Matsui Y, et al. Novel mucoadhesion tests for polymers and polymer-coated particles to design optimal mucoadhesive drug delivery systems. Adv Drug Deliv Rev. 2005;57:1583-1594.

20. Birnbaum D, Brannon-Peppas L. Microparticle Drug Delivery Systems. Totowa, NJ: Humana Press; 2003

21. Viswanathan N, Thomas P, Pandit J, et al. Preparation of non-porous microspheres with high entrapement efficiency of proteins by a (waterin-oil)-in-oil emulsion technique. J Control Release. 1999;58:9-20.

22. Weidenauer U, Bodmer D, Kissel T. Microencapsulation of hydrophilic drug substance using biodegradable polyesters. Part I: Evaluation of different techniques for the encapsulation of pamidronate disodium salt. J Microencapsul. 2003;20:509-524.

23. You S, Kwon H, Lee J, et al. Studies on the formation of hydrophobic ion-pairing complex of alendronate. Arch Pharm Res. 2009;32:1055-1060.

24. Baek J, Kwon H, Hwang J, et al. Alendronate-loaded microparticles for improvement of intestinal cellular absorption. J Drug Target. 2011;19:37-48

25. Samdancioglu S, Calis S, Summu M, et al. Formulation and in vitro evaluation of bisphosphonate loaded microspheres for implantation in osteolysis. Drug Dev Ind Pharm. 2006;32:473-481.

26. Jain R. The manufacturing techniques of various drug loaded biodegradable poly(lactide-co-glycolide)(PLGA) devices. Biomaterials. 2000;21:2475-2490.

27. Perugini $\mathrm{P}, \mathrm{Genta} \mathrm{I}$, Conti B, et al. Long-term release of clodronate from biodegradable microspheres. AAPS Pharm Sci Tech. 2001;2:E10.

28. Jang J, Kwon B, Lee H, et al. Preparation of biodegradable PLGA nanospheres employing a fast solvent evaporation method. J Ind Eng Chem. 2007;6:1043-1046.

29. Trickler W, Nagvekar A, Dash A. A novel nanoparticle formulation for sustained paclitaxel delivery. AAPS Pharm Sci Tech. 2008;9:486-493.
International Journal of Nanomedicine

\section{Publish your work in this journal}

The International Journal of Nanomedicine is an international, peerreviewed journal focusing on the application of nanotechnology in diagnostics, therapeutics, and drug delivery systems throughout the biomedical field. This journal is indexed on PubMed Central, MedLine, CAS, SciSearch ${ }^{\circledR}$, Current Contents ${ }^{\circledR} /$ Clinical Medicine,

\section{Dovepress}

Journal Citation Reports/Science Edition, EMBase, Scopus and the Elsevier Bibliographic databases. The manuscript management system is completely online and includes a very quick and fair peer-review system, which is all easy to use. Visit http://www.dovepress.com/ testimonials.php to read real quotes from published authors. 